archives-ouvertes

\title{
Scattering of guided waves from discontinuities in cylinders : numerical and experimental analysis
}

\author{
Farouk Benmeddour, Laurent Laguerre, Fabien Treyssede
}

\section{To cite this version:}

Farouk Benmeddour, Laurent Laguerre, Fabien Treyssede. Scattering of guided waves from discontinuities in cylinders: numerical and experimental analysis. International Congress on Ultrasonics, Sep 2011, France. 4 p. hal-00639669

\section{HAL Id: hal-00639669 \\ https://hal.archives-ouvertes.fr/hal-00639669}

Submitted on 9 Nov 2011

HAL is a multi-disciplinary open access archive for the deposit and dissemination of scientific research documents, whether they are published or not. The documents may come from teaching and research institutions in France or abroad, or from public or private research centers.
L'archive ouverte pluridisciplinaire HAL, est destinée au dépôt et à la diffusion de documents scientifiques de niveau recherche, publiés ou non, émanant des établissements d'enseignement et de recherche français ou étrangers, des laboratoires publics ou privés. 


\title{
Scattering of guided waves from discontinuities in cylinders: numerical and experimental analysis
}

\author{
Farouk Benmeddour*, , Laurent Laguerre* and Fabien Treyssède* \\ ${ }^{*}$ Université Nantes Angers Le Mans (LUNAM), IFSTTAR, MACS, F-44341 Bouguenais, France \\ ${ }^{\dagger} I E M N$, OAE Department, CNRS UMR 8520, University of Valenciennes and Hainaut Cambrésis, Le Mont Houy \\ 59313 Valenciennes Cedex 9, France
}

\begin{abstract}
.
The aim of this work is to study the fundamental compressional $(\mathrm{L}(0,1))$ Pochhammer-Chree mode interaction with nonaxisymmetric damages in cylinders. To this end, experimental and numerical investigations of non-axisymmetric vertical cracks are considered. A non-contact magnetostrictive device is used for experimental investigations. Magnetostrictive transducers are used to generate and receive compressional guided waves. These are enabled by using an axisymmetric and longitudinal magnetic polarising field. Both, the incident and the reflected signals are acquired by the same receiver which allows a direct calculation of the reflected power flow. Different vertical cracks with various depths milled in steel cylinders are considered. The power flows are compared with those obtained by a three dimensional numerical method. This numerical method is based on a hybrid three dimensional (3D) approach combining the classical finite element (FE) method with the semi-analytical finite element (SAFE) technique. The near field surrounding the damage is analysed with the 3D FE method whereas transparent conditions are applied to the wave guide sections for the far field analysis. These transparent conditions are based on modal expansions on cross-sections. The SAFE technique is used to compute the eigenmodes. Eigenforces and modal power flows are post-processed on a straightforward way. First, the hybrid method is validated with published results in the literature obtained for a free-end cylinder. Finally, numerical and experimental results are compared with success.
\end{abstract}

Keywords: Guided waves, cylinders, vertical cracks, 3D hybrid FE-SAFE, magnetostrictive transducers.

PACS: $43.35 . \mathrm{Cg}$, 43.35.Zc, 43.40.Le

\section{INTRODUCTION}

The interaction of guided waves with damages is a complex phenomenon and have been studied analytically [1, 2], numerically [3,4] and experimentally [5]. Analytical approaches are known to be fast from a computational point of view but generally limited to simple geometries and discontinuities. However, numerical methods allowing complexshaped waveguides or damage geometries are very time consuming. Hybrid approaches, combining a FE method and the normal mode expansion technique have been developed for studying wave scattering by defects in plates [6,7]. The modal decomposition performed both at the inlet and outlet of a waveguide FE model, by using analytical solutions for the guided modes of the considered structure which, can then be limited to a small region surrounding the defect. The scattered solution is computed for each frequency and yields directly the coefficients of scattered modes without any post-processing. Analytical solutions for guided modes have been replaced in several occasions by the semi-analytical finite element (SAFE) technique (see e.g. Refs. $[8,9]$ ) to give rise to the so-called hybrid FE-SAFE method capable of handling arbitrary cross-sections with complex-shaped defects (see for instance $[10,11,12]$ ). In the first part, we present a study of the interaction of guided waves with non-axisymmetric cracks in infinite circular cylinders.It is based on the development of a general 3D hybrid FE-SAFE procedure. A 3D variational formulation of the FE method is used and combined with a normal mode expansion applied to the cross-sections of the cylindrical waveguide which enables the separation between ingoing and outgoing modes. The SAFE technique is used to compute the eigenvectors of the eignmodes. Then, forces are derived directly from the SAFE matrices as well as power reflection and transmission coefficients. This allows to dispense with the tedious step of post-processing for computing consistent load eigenvectors. In the second part, the 3D hybrid FE-SAFE predictions are compared to experimental data for the reflection of the $(\mathrm{L}(0,1))$ fundamental Pochhammer-Chree mode from non-axisymmetric vertical cracks. These are achieved for various depths using a pulse echo magnetostrictive device. 


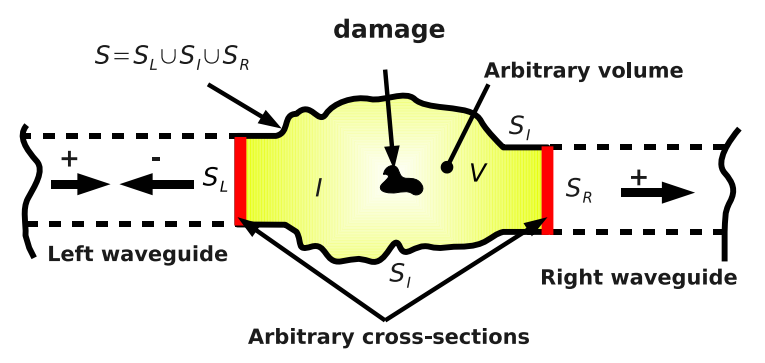

FIGURE 1. Description of the problem.

\section{HYBRID FE-SAFE METHOD}

\section{General description of the problem}

Figure 1 depicts a damage located inside an arbitrary volume $V$ connected to two semi-infinite arbitrary crosssection waveguides. The interior region is connected to the left and right waveguides through boundaries denoted $S_{L}$ and $S_{R}$, respectively. The volume is bounded by $S_{L}$ and $S_{R}$ and by a traction free boundary $S_{I}$. In a first step, for the volume $V$, a standard FE method with a time harmonic regime is used [13]:

$$
\int_{V} \delta \boldsymbol{\varepsilon}^{T} \boldsymbol{\sigma} d V-\omega^{2} \int_{V} \rho \delta \mathbf{u}^{T} \mathbf{u} d V-\int_{S} \delta \mathbf{u}^{T} \mathbf{t} d S=0
$$

The classical FE discretised variational formulation can be partitioned on the volume $V$ as follows (here interior forces are not considered) :

$$
\left\{\begin{array}{c}
\delta \mathbf{U}_{L} \\
\delta \mathbf{U}_{I} \\
\delta \mathbf{U}_{R}
\end{array}\right\}^{T}\left[\begin{array}{lll}
\mathbf{D}_{L L} & \mathbf{D}_{L I} & \mathbf{D}_{L R} \\
\mathbf{D}_{I L} & \mathbf{D}_{I I} & \mathbf{D}_{I R} \\
\mathbf{D}_{R L} & \mathbf{D}_{R I} & \mathbf{D}_{R R}
\end{array}\right]\left\{\begin{array}{c}
\mathbf{U}_{L} \\
\mathbf{U}_{I} \\
\mathbf{U}_{R}
\end{array}\right\}-\left\{\begin{array}{c}
\delta \mathbf{U}_{L} \\
\delta \mathbf{U}_{I} \\
\delta \mathbf{U}_{R}
\end{array}\right\}^{T}\left\{\begin{array}{c}
\mathbf{f}_{L} \\
\mathbf{0} \\
\mathbf{f}_{R}
\end{array}\right\}=0
$$

where $\mathbf{U}_{L}, \mathbf{U}_{I}$ and $\mathbf{U}_{R}$ respectively denote the left, interior and right displacement dofs. $\mathbf{D}_{i j}=\mathbf{K}_{i j}-\omega^{2} \mathbf{M}_{i j}(i, j=L, I, R)$ are the associated partitions related to stiffness and mass quantities.f represents external time harmonic forces. $\delta(\cdot)$ denotes virtual fields, $\omega$ is the angular frequency. Then, on the waveguide cross-sections, the displacements and forces are expanded into sums of modes. On $S_{L}$, the sum is decomposed into ingoin and outgoing modes. On $S_{R}$, only the outgoing modes are taken into account which enforces the coefficients of ingoing modes to zero giving rise to a transparent boundary condition. Displacements and forces on the left and right sections are then given by:

$$
\mathbf{U}_{L}=\sum_{n=1}^{N_{L}^{+}} \alpha_{L n}^{+} \mathbf{U}_{L n}^{+}+\sum_{n=1}^{N_{L}^{-}} \alpha_{L n}^{-} \mathbf{U}_{L n}^{-}, \mathbf{f}_{L}=\sum_{n=1}^{N_{L}^{+}} \alpha_{L n}^{+} \mathbf{f}_{L n}^{+}+\sum_{n=1}^{N_{L}^{-}} \alpha_{L n}^{-} \mathbf{f}_{L n}^{-}, \mathbf{U}_{R}=\sum_{n=1}^{N_{R}^{+}} \alpha_{R n}^{+} \mathbf{U}_{R n}^{+}, \mathbf{f}_{R}=\sum_{n=1}^{N_{R}^{+}} \alpha_{R n}^{+} \mathbf{f}_{R n}^{+},
$$

where $n$ denotes the mode number, $N_{L}^{ \pm}$and $N_{R}^{+}$are the number of modes considered in the expansions. $\alpha_{L n}^{ \pm}(n=$ $\left.1, \ldots, N_{L}^{ \pm}\right)$and $\alpha_{R n}^{+}\left(n=1, \ldots, N_{R}^{+}\right)$are the left and right displacement modal amplitudes, respectively. Eqs. (3) can be transformed into products of matrices. For the left section, coefficients of positive-going modes are imposed so that $\delta \alpha_{L n}^{+}=0$. Displacements, forces and virtual displacements can then be expressed as:

$$
\begin{aligned}
& \mathbf{U}_{L}=\mathbf{B}_{L}^{+} \boldsymbol{\alpha}_{L}^{+}+\mathbf{B}_{L}^{-} \boldsymbol{\alpha}_{L}^{-}, \mathbf{f}_{L}=\mathbf{T}_{L}^{+} \boldsymbol{\alpha}_{L}^{+}+\mathbf{T}_{L}^{-} \boldsymbol{\alpha}_{L}^{-}, \delta \mathbf{U}_{L}=\delta \boldsymbol{\alpha}_{L}^{-T} \mathbf{B}_{L}^{-T} \\
& \mathbf{U}_{R}=\mathbf{B}_{R}^{+} \boldsymbol{\alpha}_{R}^{+}, \mathbf{f}_{R}=\mathbf{T}_{R}^{+} \boldsymbol{\alpha}_{R}^{+}, \delta \mathbf{U}_{R}=\delta \boldsymbol{\alpha}_{R}^{+T} \mathbf{B}_{R}^{+T}
\end{aligned}
$$

For any trial field $\delta \boldsymbol{\alpha}_{L}^{-}, \delta \mathbf{U}_{I}$ and $\delta \boldsymbol{\alpha}_{R}^{+}$, and after rearrangements one can obain the following linear global system:

$$
\begin{aligned}
{\left[\begin{array}{ccc}
\mathbf{B}_{L}^{-T} & \mathbf{0} & \mathbf{0} \\
\mathbf{0} & \mathbf{I} & \mathbf{0} \\
\mathbf{0} & \mathbf{0} & \mathbf{B}_{R}^{+T}
\end{array}\right] } & \left(\left[\begin{array}{ccc}
\mathbf{D}_{L L} & \mathbf{D}_{L I} & \mathbf{D}_{L R} \\
\mathbf{D}_{I L} & \mathbf{D}_{I I} & \mathbf{D}_{I R} \\
\mathbf{D}_{R L} & \mathbf{D}_{R I} & \mathbf{D}_{R R}
\end{array}\right]\left[\begin{array}{ccc}
\mathbf{B}_{L}^{-} & \mathbf{0} & \mathbf{0} \\
\mathbf{0} & \mathbf{I} & \mathbf{0} \\
\mathbf{0} & \mathbf{0} & \mathbf{B}_{R}^{+}
\end{array}\right]-\left[\begin{array}{ccc}
\mathbf{T}_{L}^{-} & \mathbf{0} & \mathbf{0} \\
\mathbf{0} & \mathbf{0} & \mathbf{0} \\
\mathbf{0} & \mathbf{0} & \mathbf{T}_{R}^{+}
\end{array}\right]\right)\left\{\begin{array}{c}
\boldsymbol{\alpha}_{L}^{-} \\
\mathbf{U}_{I} \\
\boldsymbol{\alpha}_{R}^{+}
\end{array}\right\} \\
& =-\left[\begin{array}{ccc}
\mathbf{B}_{L}^{-T} & \mathbf{0} & \mathbf{0} \\
\mathbf{0} & \mathbf{I} & \mathbf{0} \\
\mathbf{0} & \mathbf{0} & \mathbf{B}_{R}^{+T}
\end{array}\right]\left(\left[\begin{array}{ccc}
\mathbf{D}_{L L} & \mathbf{D}_{L I} & \mathbf{D}_{L R} \\
\mathbf{D}_{I L} & \mathbf{D}_{I I} & \mathbf{D}_{I R} \\
\mathbf{D}_{R L} & \mathbf{D}_{R I} & \mathbf{D}_{R R}
\end{array}\right]\left[\begin{array}{c}
\mathbf{B}_{L}^{+} \\
\mathbf{0} \\
\mathbf{0}
\end{array}\right]-\left[\begin{array}{c}
\mathbf{T}_{L}^{+} \\
\mathbf{0} \\
\mathbf{0}
\end{array}\right]\right) \boldsymbol{\alpha}_{L}^{+} .
\end{aligned}
$$


The unknown vectors $\boldsymbol{\alpha}_{L}^{-}, \mathbf{U}_{I}$ and $\boldsymbol{\alpha}_{R}^{+}$are found by solving the above linear system at each frequency.

In a second step, the SAFE method is used in order to determine the elastic guided modes for the left and right cross-sections of the interior region. This technique reduces the analysis of three dimensional waveguides to two dimensions by using a spatial Fourier transform along the propagation direction $\left(\mathbf{u}(x, y, z)=\mathbf{u}(x, y) e^{+j k z}\right.$, where $k$ is the wavenumber and $z$ is the propagation direction). Only the cross-sections have to be meshed, allowing a fast and accurate computation of eigenmodes for any arbitrary cross-section. The SAFE variational formulation is written as:

$$
\delta \mathbf{U}\left(\mathbf{K}_{1}-\omega^{2} \mathbf{M}_{S}-j k\left(\mathbf{K}_{2}-\mathbf{K}_{2}^{T}\right)+k^{2} \mathbf{K}_{3}\right) \mathbf{U}=0
$$

obtained from the following elementary matrices: $\mathbf{K}_{1}^{e}=\int_{S^{e}} \mathbf{B}_{1}^{T} \mathbf{C B}_{1} d S, \mathbf{K}_{3}^{e}=\int_{S^{e}} \mathbf{B}_{2}^{T} \mathbf{C B}_{2} d S$ and $\mathbf{K}_{2}^{e}=\int_{S^{e}} \mathbf{B}_{2}^{T} \mathbf{C B}_{1} d S$, $\mathbf{M}_{S}^{e}=\int_{S^{e}} \rho \mathbf{N}^{e T} \mathbf{N}^{e} d S$, where $\mathbf{B}_{1}=\mathbf{L}_{x} \mathbf{N}_{, x}^{e}+\mathbf{L}_{y} \mathbf{N}_{, y}^{e}$ and $\mathbf{B}_{2}=\mathbf{L}_{z} \mathbf{N}^{e} . \mathbf{N}_{, x}^{e}, \mathbf{N}_{, y}^{e}, \mathbf{L}_{x}, \mathbf{L}_{y}$ and $\mathbf{L}_{z}$ are defined in a previous work [13]. By fixing the angular frequency $\omega$ and finding the wavenumber $k$, Eq. (6) leads to a quadratic eigenvalue problem. The resolution of this system, after linearisation, for the left (right) cross-section gives wavenumbers $k_{L n}$ $\left(k_{R n}\right)$ and modeshapes $\mathbf{U}_{L n}\left(\mathbf{U}_{R n}\right)$. The separation into ingoing and outgoing modes then yields the displacement modal bases $\mathbf{B}_{L, R}^{ \pm}$used in the previous subsection. The next step is to determine the modal bases of forces $\mathbf{T}_{L, R}^{ \pm}$. The idea is to derive forces directly from SAFE matrices. For clarity, let us first consider the right cross-section. From the third left integral of Eq. (1) and the application of normal mode expansions, forces on the right section can be rewritten as: $\delta \mathbf{U}_{R}^{T} \mathbf{f}_{R}=\int_{S_{R}} \delta \mathbf{u}^{T}\left(\sum_{n=1}^{N_{R}^{+}} \alpha_{R n}^{+} \mathbf{t}_{R n}^{+}\right) d S$. For one element of surface, $\delta \mathbf{u}^{T}=\delta \mathbf{U}^{e^{T}} \mathbf{N}^{e^{T}}$. Following the approach as in Treyssède [14] for computing the energy velocity, we verified that $\mathbf{t}_{R n}^{+}=\mathbf{L}_{z}^{T} \boldsymbol{\sigma}_{R n}^{+}=\mathbf{L}_{z}^{T} \mathbf{C}\left(\mathbf{B}_{1}+j k_{R n}^{+} \mathbf{B}_{2}\right) \mathbf{U}_{R n}^{+e}$, which yields: $\delta \mathbf{U}_{R}^{T} \mathbf{f}_{R}=\delta \mathbf{U}_{R}^{T} \sum_{n=1}^{N_{R}^{+}} \alpha_{R n}^{+}\left(\mathbf{K}_{2}+j k_{R n}^{+} \mathbf{K}_{3}\right) \mathbf{U}_{R n}^{+}$. This result defines explicitly the forces $\mathbf{f}_{R n}^{+}$, involved inside the modal basis $\mathbf{T}_{R}^{+}$as: $\mathbf{f}_{R n}^{+}=\left(\mathbf{K}_{2}+j k_{R n}^{+} \mathbf{K}_{3}\right) \mathbf{U}_{R n}^{+}$, where $\mathbf{K}_{2}$ and $\mathbf{K}_{3}$ are the SAFE matrices associated with the right section. Once the displacement modal bases are obtained, the calculation of modal bases $\mathbf{T}_{L}^{ \pm}$and $\mathbf{T}_{R}^{+}$is then direct and consistent with the used FE approximation. For hybrid FE-SAFE methods, this may simplify greatly the tedious postprocess of consistent loads based on displacement derivatives associated with each mode. In the final step, the power reflection and transmission coefficients are computed by dividing the reflected and transmitted powers by the incident power, respectively: $R_{m n}=\frac{P_{L n}^{-}}{P_{L m}^{+}}, T_{m n}=\frac{P_{R n}^{+}}{P_{L m}^{+}}$, where $m$ is the incident mode, $n$ is the reflected or transmitted mode, and $P_{L m}^{+}, P_{L n}^{-}$and $P_{R n}^{+}$are computed with derived equations from Poynting vector definition and Auld's [1] orthogonality relationship.

\section{EXPERIMENTAL RESULTS}

Experimental tests were carried out in order to validate the simulation results relative to the sensitivity of the axisymmetric $\mathrm{L}(0,1)$ mode to vertical notches in a cylinder. Simple vertical notches were progressively created with a hand saw to the depths of $0.4,1.2,2.2,3.5,4.2 \mathrm{~mm}$ until complete cut in a steel cylinder specimen of $5.4 \mathrm{~mm}$ in diameter and length $\mathrm{L}$ of $6 \mathrm{~m}$. The time history signal of the pulse propagating along the cylinder was recorded for each notch depth. Time domain measurements were performed in pulse-echo using non-contact magnetostrictive transducers $[15,16]$. The magnetostrictive device consists of two small encircling dynamic coils for the emission and reception, respectively, and each coil is mounted coaxially with a solenoid. The magnetic polarizing field strength imposed along the longitudinal direction by the solenoid allows the device to excite and detect longitudinal guided waves. The magnetostrictive transducers operate in the low-frequency range (here $\Omega$ is lower than 1.5). The geometrical arrangement of the non-contact transducers allows systematic recordings of both the incident and reflected waves for each notch depth. In order to obtain time-resolved echoes (i.e. no interference between echoes from the defect and from the ends of the cylinder), the defect, the transmitter and the detector are positioned at 5L/8, L/4 and L/2 respectively. This gives notch-to-transducer distances respectively of $3 \mathrm{~L} / 8$ for the transmitter and $\mathrm{L} / 8$ for the detector respectively. Excitation of the $\mathrm{L}(0,1)$ mode was achieved by driving the transmitter with a ten cycle gaussian-shaped tone-burst whose maximum frequency is lower than the cut-off frequency of the $\mathrm{L}(0,2)$ mode. The excitation center-frequencies were varied between 100 to $230 \mathrm{kHz}$ by $10 \mathrm{kHz}$ steps. The fourteen time domain signals were then processed to extract the reflection coefficient in the frequency domain. Here, the $\mathrm{L}(0,1)$ mode reflection coefficient was derived by dividing the spectral component of the $\mathrm{L}(0,1)$ reflected pulse by the spectral component of the $\mathrm{L}(0,1)$ incident pulse at the center-frequency of the excitation pulse. Figure 2a presents the modulus of the reflection coefficient of the $\mathrm{L}(0,1)$ 

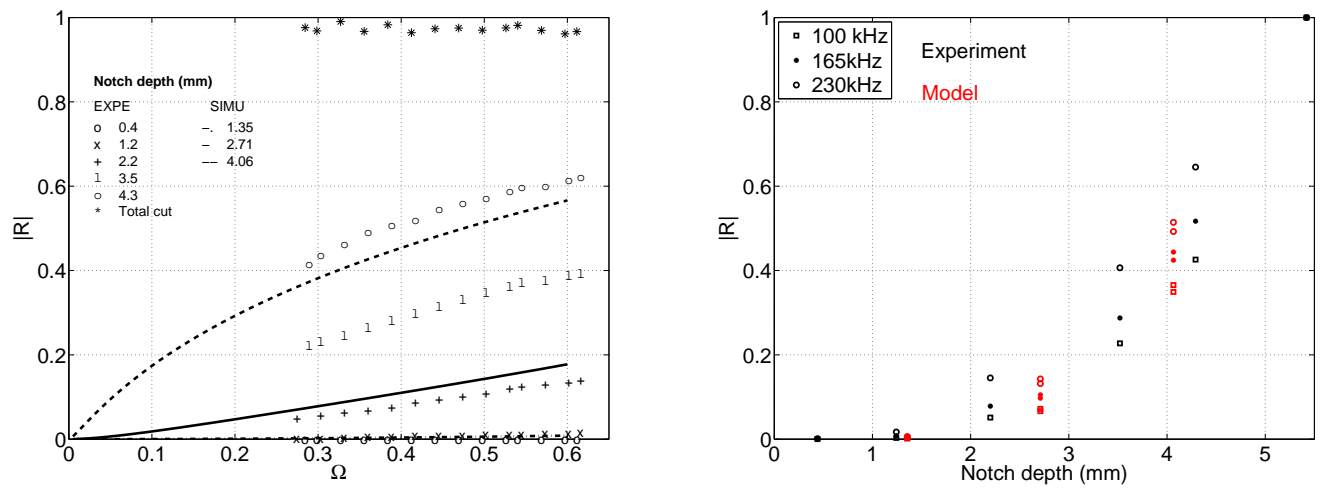

FIGURE 2. Experimental and simulated $\mathrm{L}(0,1)$ reflection coefficients as a function of (a) normalised frequency for various notch depths and (b) the notch depth for the low-, mid- and high-center frequencies.

mode as a function of the normalised frequency both for the experimental and simulated datasets. Simulations are done for a Poisson's coefficient of 0.31 . This first experimental to theoretical comparison is fairly good since first, both datasets exhibit the same trends versus frequency and second, the relative position of theoretical to experimental data versus the notch depth is respected. This encouraging results are confirmed by those presented in figure $2 \mathrm{~b}$. Figure $2 \mathrm{~b}$ shows the good agreement between the simulated and experimental reflection coefficients for the $\mathrm{L}(0,1)$ mode versus the notch depth. Double-valued simulation results are obtained for two Poisson's ratios of 0.25 and 0.31 (the higher reflection coefficient value is associated to the higher Poisson's ratio).

\section{CONCLUSIONS}

In this paper, a 3D hybrid numerical method has been developed for the investigation of wave scattering in elastic waveguides. This method combines the classical FE method and the so-called SAFE technique. It has the main advantage of being able to handle complex-shape inhomogeneities in waveguides of arbitrary cross-sections. Moreover, it gives a formula that renders straightforward the calculation of eigenforces and modal power flows. A preliminary experimental validation of the hybrid 3D method has been done for vertical saw-cuts of different depths. The first comparisons of the theoretical/experimental reflection coefficient, for the $(\mathrm{L}(0,1))$ Pochammer-Chree mode, as a function of normalized frequency or notch depth are encouraging.

\section{REFERENCES}

1. B. Auld, Acoustic fields and waves in solids, vol. II, A Wiley-Interscience publication, 1973.

2. G. Shkerdin, and C. Glorieux, J. Acoust. Soc. Am. 116 (4), 2089-2100 (2004).

3. F. Benmeddour, S. Grondel, J. Assaad, and E. Moulin, NDT \& E International 41, 1-9 (2008).

4. F. Benmeddour, S. Grondel, J. Assaad, and E. Moulin, NDT \& E International 41, 330-340 (2008).

5. F. Benmeddour, S. Grondel, J. Assaad, and E. Moulin, Ultrasonics 49, 202-205 (2009).

6. W. Karunasena, A. Shah, and S. Datta, Journal of Engineering Mechanics 117, 1738-1754 (1991).

7. Y. Al-Nassar, S. Datta, and A. Shah, Ultrasonics 29, 125-132 (1991).

8. V. Damljanovic, and R. L. Weaver, J. Acoust. Soc. Am. 115, 1572-1581 (2004).

9. T. Hayashi, W.-J. Song, and J. Rose, Ultrasonics 41, 175-183 (2003).

10. S. Datta, A. Shah, and W. Karunasena, Mechanics of Composite Materials and Structures 6, 285-300 (1999).

11. N. Rattanawangchroen, W. Zhuang, A. Shah, and S. Datta, Journal of Engineering Mechanics pp. 1020-1026 (1997).

12. J. Galan, and R. Abascal, Int. J. Numer. Meth. Engng 58, 1091-1118 (2003).

13. F. Benmeddour, F. Treyssède, and L. Laguerre, International Journal of Solids and Structures 48, 764 - 774 (2011).

14. F. Treyssède, Wave Motion 45, 457-470 (2008).

15. L. Laguerre, J.-C. Aime, and M. Brissaud, Ultrasonics 39 (7), 503-514 (2002).

16. J.-C. Aime, M. Brissaud, and L. Laguerre, J. Acoust. Soc. Am. 109 (1), 51-58 (2001). 